Ann. Biol. anim. Bioch. Biophys., I970, 10 (I), I5-24.

\title{
ÉTUDE DU MOMENT D'OVULATION CHEZ LA BREBIS NORMALE OU TRAITÉE PAR UN PROGESTAGÈNE ASSOCIÉ OU NON A UNE INJECTION DE PMSG
}

\author{
Y. COGNIÉ, J.-C. MARIANA et J. THIMONIER \\ Laboratoire de Physiologie de la Reproduction, \\ Centre de Recherches de Tours, 37 - Nouzilly \\ Institut national de la Recherche agronomique
}

\section{RÉSUMF́}

Le moment d'ovulation a été étudié pendant l'anœstrus saisonnier et pendant la saison sexuelle chez des brebis normales ou traitées par un progestagène associé ou non à une injection intramusculaire de PMSG.

Chez les brebis traitées, PMSG diminue l'intervalle moyen " arrêt du traitement - apparition de l'œestrus ". L'étalement de l'apparition des œstrus est également réduit. Pendant la saison sexuelle, après arrêt du traitement, les œstrus apparaissent plus rapidement que pendant l'anœstrus saisonnier.

Chez les brebis normales, c'est à $I 7$ heures que le plus grand nombre d'animaux vient pour la première fois en œestrus. Une injection de PMSG faite le matin à 9 heures lors de l'arrêt du traitement accentue cet effet du rythme nycthéméral.

Pour les brebis recevant un progestagène, il ne semble pas exister une relation directe entre la fin du traitement et le moment d'ovulation.

L'instant moyen d'ovulation calculé après analyse par la méthode des probits se situe respectivement pour les brebis témoins et les brebis recevant le progestagène seul, $32 \mathrm{~h} o \pm 0,8 \mathrm{I}$ et 3o $h 4 \pm$ r,33 après le début observé de l'œestrus. Ces deux valeurs ne sont pas différentes statistiquement.

Pour les brebis recevant PMSG, il semble exister deux groupes d'animaux :

- un premier groupe représentant $20 \mathrm{p}$. roo de l'échantillon examiné et dont la réponse est rapide puisque l'ovulation est effective moins de 20 heures après le début observé de l'œstrus;

- un deuxième groupe dont le moment moyen d'ovulation se situe à $29 \mathrm{~h} 8 \pm 0,49$ après le début observé de l'œstrus, valeur non différente statistiquement de celles trouvées pour les brebis témoins et les brebis traitées avec le progestagène seul. Une injection intramusculaire de PMSG modifie donc le moment d'ovulation chez une partie seulement des animaux traités. 


\section{INTRODUCTION}

Par suite de 1'utilisation, lors de l'insémination artificielle ovine, d'un faible nombre de spermatozoïdes, le moment du dépôt de ceux-ci dans les voies génitales femelles par rapport à celui de l'ovulation est important pour l'obtention d'un taux de fécondation maximum. Cette importance a été montrée en particulier par les résultats obtenus par insémination artificielle après synchronisation des œestrus par les progestagènes (Colas et CogNié, I968).

Au cours de cycles œestriens normaux, l'ovulation se produit en général de 25 à 30 heures après le début observé de l'œestrus (ZELTOBRUkH et RAK, I964; PARsons, Hunter et RAyNER, I967) ou vers la fin de l'œstrus (MCKENZIE et TERRIL,L, I937) bien que l'ovulation puisse avoir lieu plusieurs heures avant ou plusieurs heures après la fin de l'œestrus. Après traitements progestatifs à l'aide d'éponges vaginales (Robinson, I965), Robinson et SMITh (I967) n'ont pas trouvé de modification du moment de l'ovulation par rapport au début de l'œstrus. Cependant, en période d'anœstrus saisonnier ou chez des brebis allaitantes, il est nécessaire d'ajouter une injection de PMSG au traitement progestatif pour induire régulièrement l'œstrus et 1'ovulation (THIMONIER et al., Ig68). Aussi avons-nous repris l'étude du moment d'ovulation dans ce dernier cas, l'injection de PMSG étant susceptible d'apporter des modifications.

\section{MATÉRIEL ET MÉTHODES}

\section{r. Animaux et traitements}

Quatre-vingt-dix-huit brebis de race Ile-de-France âgées de 2 à 7 ans ont été utilisées à deux périodes différentes de l'année.

Des éponges en polyuréthane imprégnées de $40 \mathrm{mg}$ d'acétate de fluorogestone (RoBINson, rg65) ont été mises en place pour une durée de I4 jours dans le vagin des brebis. La pose et le retrait des éponges ont été effectuées le matin entre 9 heures et $9 \mathrm{~h} 30$.

a) Anosirus saisonnier.

Les animaux ont été divisés en deux lots traités à 2 semaines d'intervalle (retrait des éponges le I6 avril I968 et le 30 avril I968), a fin de permettre une meilleure détection de l'oestrus, l'activité sexuelle des béliers étant faible à cette période de l'année.

Quarante-neuf brebis ( 25 le $16-4$ et 24 le $30-4$ ) ont reçu uniquement le traitement progestatif.

Quarante-neuf brebis réparties de la même façon ont reçu une injection intramusculaire de 800 UI de PMSG au moment du retrait de l'éponge vaginale. Cette dose d'hormone gonadotrope sérique est celle qui est administrée aux brebis lors des expériences de lutte provoquée en période d'ancestrus.

\section{b) Début de saison sexuelle.}

Pour la totalité des mêmes brebis, le retrait de l'éponge vaginale a eu lieu le 2 septembre I 968 entre 9 heures et 9 h 30 .

Quarante-neuf brebis ont reçu une injection intramusculaire de PMSG au moment du retrait de l'éponge vaginale. Afin d'éviter une superovulation importante, la dose de PMSG a été volontairement limitée à $400 \mathrm{LI}$.

Les brebis n'étant pas inséminées, les œstrus sont réapparus $r 7$ jours environ après l'cestrus induit par le traitement. Ces brebis ont constitué alors un lot témoin pour l'étude du moment d'ovulation. 


\section{Détection de l'cestrus}

Elle a été effectuée toutes les 4 heures, après l'enlèvement des éponges, selon la méthode décrite par Mauléon et Dauzier (I965). Le chevauchement de la femelle par le bélier, et l'immobilité de la femelle ont été les seuls critères d'œstrus retenus.

Des béliers de races Limousine et Préalpes ont été utilisés pour la détection de l'œestrus.

Les brebis ont été retirées au fur et à mesure de leur détection et ont été séparées des mâles, la présence de ceux-ci pouvant modifier le moment d'ovulation (PARsons, HuNTER et RAYNER, Ig67).

\section{Contrôle de l'ounlation}

Les brebis traitées en œstrus ont été réparties en cinq lots pour le contrôle de l'ovulation qui a été fait 20,23,26, 29 ou 32 heures après le début observé de l'œstrus. Les brebis témoins (septembre 1968) ont également été réparties en cinq lots pour le contrôle de l'ovulation qui a été effectué $23,26,29,32$ ou 35 heures après le début observé de l'œestrus. La modification des moments de contrôle a été faite après l'obtention des résultats chez les brebis traitées.

Chaque brebis n'a subi qu'un seul contrôle ovarien. Cette opération faite par colioscopie (Thimonier et Mauléon, ig67 et ig69) sous anesthésie générale n'entraîne pas d'éclatement des follicules, l'observation se faisant in situ sans qu'il soit nécessaire de manipuler les ovaires euxmêmes.

L'opération peut être effectuée sur des brebis non anesthésiées. Il est alors possible de faire plusieurs contrôles sur une même brebis. Nous avons cependant rejeté cette possibilité. Sans anesthésie générale, le tonus musculaire de l'utérus est en effet trop élevé et les manipulations nécessaires pour l'observation des ovaires peuvent alors provoquer un éclatement accidentel des follicules.

\section{Analyse statistique}

La méthode des probits a été utilisée (FINNEY, I962). Cette méthode permet l'analyse d'essais à réponse qualitative du type " proportion d'animaux ayant ou n'ayant pas ovulé après un traitement donné n.

Elle consiste en une transformation des pourcentages cumulés d'une loi normale en valeurs appelées probits ce qui transforme la courbe sigmoïde en droite ; par itération, à partir des valeurs initiales des probits, nous avons calculé la valeur moyenne du paramètre telle que $5^{\circ}$ p. Ioo des animaux ont répondu, ainsi que l'écart-type de cette valcur.

\section{RÉSULTATS}

\section{Apparition de l'astrus}

a) Influence de PMSG.

Quelle que soit la dose injectée (400 UI ou 800 UI) PMSG réduit l'intervalle moyen fin de traitement-début observé de l'œstrus.

Cet intervalle est de $3 \mathrm{I}_{\mathrm{h}} 4 \pm 0,5^{6}$ pour les brebis recevant PMSG et de 39 h $9 \pm 0,90$ pour les animaux traités avec le progestagène seul.

L'étalement de l'apparition des œestrus est également diminué. Plus de 95 p. Ioo des brebis recevant PMSG viennent en œestrus en I 6 heures, alors qu'il faut 24 heures pour arriver au même résultat chez les brebis recevant le progestagène seul (fig. I).

\section{b) Infuence de la saison.}

\section{Cette influence est double:}

Io Pendant la saison sexuelle, le pourcentage de brebis traitées avec l'acétate de fluorogestone seul venant en œstrus ( $98 \mathrm{p}$. Ioo) est plus élevé que pendant la période d'anœstrus (80,4 p. I00). Cette différence est statistiquement significative: $(\mathrm{P}<0,05)$. 
$2^{\circ}$ L'intervalle arrêt du traitement-apparition des chaleurs est plus court pendant la saison sexuelle que pendant la période d'anoestrus, que les brebis reçoivent ou non une injection intramusculaire de PMSG (fig. I et tab1. I).

\section{TABLEAU I}

Influence de la saison sur l'intervalle moyen fin du traitement progestatif-début observé des chaleurs

\begin{tabular}{|c|c|c|}
\hline Traitement & Anoestrus & Saison sexuelle \\
\hline ligA seul & 42h & 37 h $\underset{(48)}{7 \pm} 1,09$ \\
\hline $\mathrm{FGA}+\mathrm{PMSG}$ & $32 \mathrm{~h} 7 \underset{(48)}{ }$ & $\begin{array}{c}30 \mathrm{~h} 0 \pm 0,80 \\
(49)\end{array}$ \\
\hline
\end{tabular}

( ) Nombre d'animaux venus en cestrus.

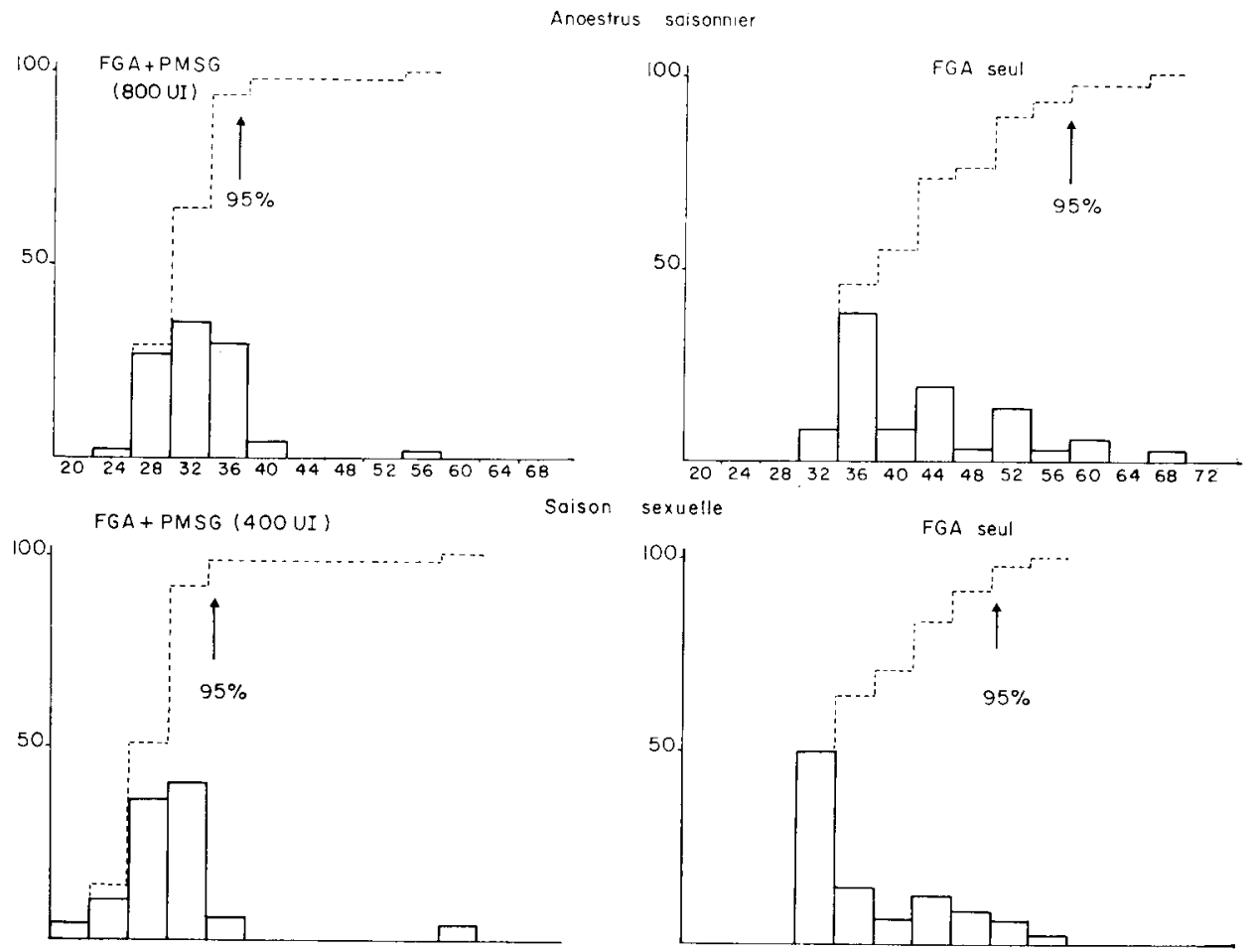

Intervalle retrait de l'éponge - apporition de l'oestrus (noures)

FIG. I. - Apparition de l'cestrus après traitement progestatif associé ou non a PMSG. Influence de la saison 
c) Apparition des cestrus au cours de la journée (fig. 2).

89,7 p. Ioo des brebis recevant une injection de PMSG (800 ou 400 UI) ont été détectées pour la première fois en œstrus entre 13 heures et 22 heures.
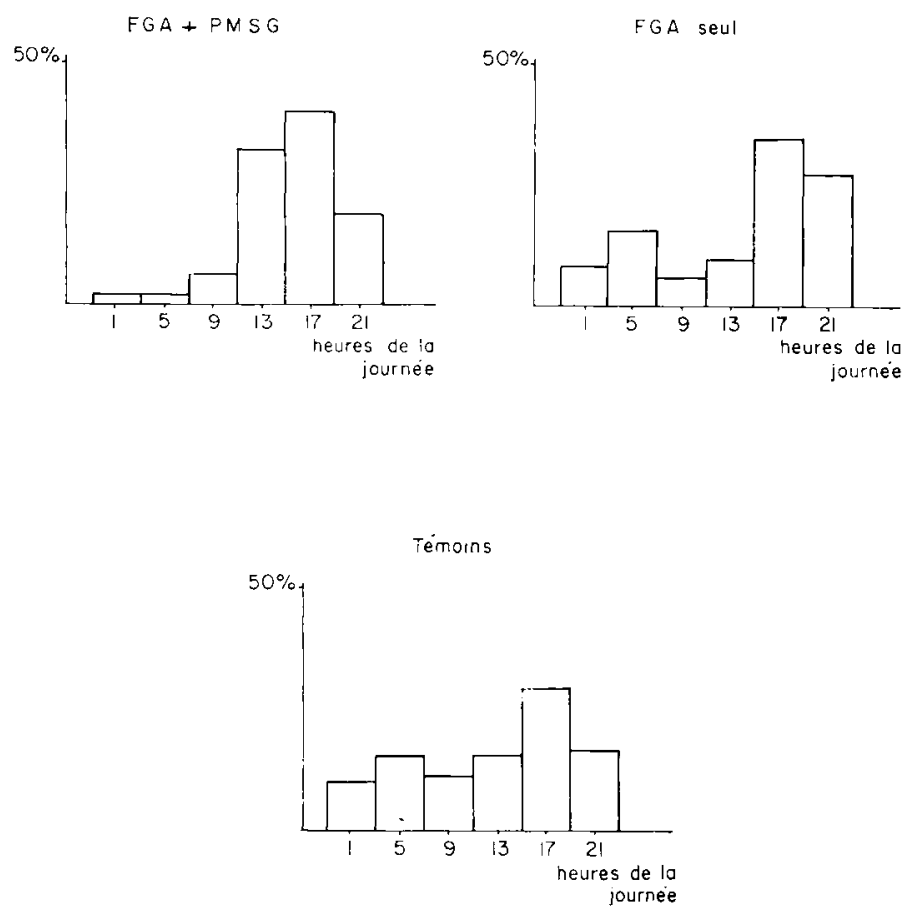

FIG. 2. - Apparition de l'cestrus au cours de la journée

Pendant la même période de la journée, 70,6 p. Ioo des brebis ne recevant que le progestagène et 62 , I p. roo des brebis témoins sont venues en cestrus. Pour ces trois catégories d'animaux c'est à $\mathrm{I} 7$ heures que le plus grand nombre de brebis a été détecté en cestrus pour la première fois.

d) Intervalle fin du traitement progestatif-apparition des retours en cestrus.

Les premiers retours en œstrus sont apparus 17 jours après le retrait des éponges vaginales. $9,3 \mathrm{p}$. Ioo des brebis sont venues en œestrus le I $8 \mathrm{e}$ jour, 46,4 p. Ioo le I $9^{\mathrm{e}}$ jour, $32, \mathrm{o}$ p. Ioo le $2 \mathrm{O}^{\mathrm{e}}$ jour et $9,3 \mathrm{p}$. Ioo le $2 \mathrm{I}^{\mathrm{e}}$ jour après le retrait des éponges vaginales. Il n'apparaît pas de différence entre les brebis ayant reçu une injection de PMSG et celle ayant été traitées uniquement au progestagène.

\section{Moment de l'ovulation}

Chez les femelles traitées, il ne semble pas qu'il y ait une relation directe entre la fin du traitement et le moment de l'ovulation. 
Bien que la saison ait une influence sur l'apparition de l'œstrus, elle ne modifie pas le moment de l'ovulation par rapport au début observé de l'œstrus. De même, il n'existe pas de différence pour le moment de l'ovulation entre les deux doses de PMSG (400 UI o1 $800 \mathrm{UI}$ ).

Seules trois catégories d'animaux ont donc été considérées sans tenir compte de la saison ou de la différence entre les closes de PMISG et l'étude du moment d'ovulation a été faite par rapport au début observé de l'ostrus.

- brebis témoins,

- brebis recevant le progestagène seul,

- brebis recevant le progestagène et une injection intramusculaire de PMSG.

A. - Pour les deux premières catégories d'animaux aucune brebis n'a ovulé 20 heures après le début observé de l'œstrus. 32 heures après, 55,6 p. Ioo et 56,2 p. Ioo seulement des brebis ont ovulé (tab1. 2).

La transformation des pourcentages cumulés en probits permet d'obtenir une droite (fig. 3). Il est donc possible de calculer le moment moyen d'ovulation (5o p. Ioo des brebis ayant ovulé). Ces moments se situent respectivement à 32 ho 00,8 I pour les brebis témoins et à $30 \mathrm{~h} 4 \pm \mathrm{I}, 33$ pour les brebis recevant le progestagène seul. La différence entre les deux moments moyens calculés d'ovulation n'est pas statistiquement significative.

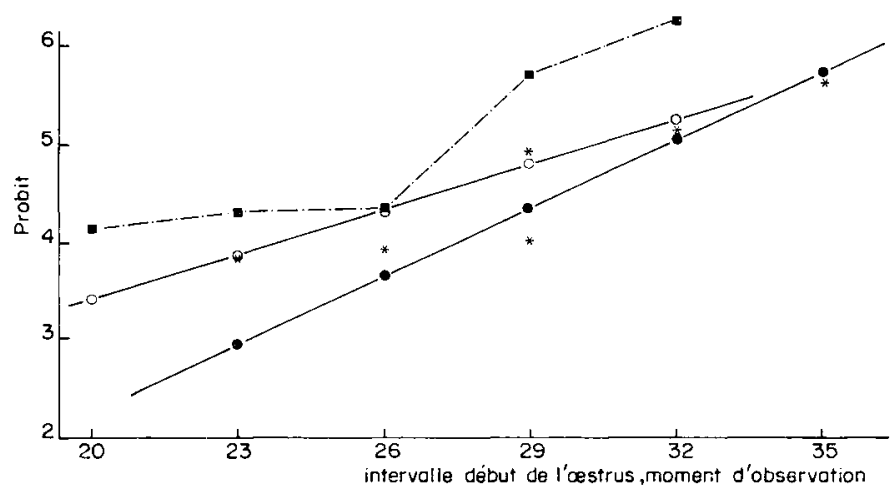

FIG. 3. - Transformation probit des pourcentages des brebis ayant ovulé à différents moments après le début observé de l'cestrus

I'ajustement linéaire est satisfaisant pour les brebis témoins (•) et les brebis recevant le progestagène seul (O).

Il ne peut être effectué dans le cas des brebis recevaut le progestagène et une injection intramusculaire PMSG (a).

* Valeur tabulée cles profits.

B. - Pour les brebis recevant une injection complémentaire de PMSG lors du retrait de l'éponge vaginale, l'ovulation semble commencer plus tôt après le début observé de l'œstrus : 2 I, I p. Ioo des brebis ont déjà ovulé moins de 20 heures après le début de l'œstrus et il en est de même pour 90 p. Ioo des brebis examinées 32 heures après le commencement de 1'œstrus (tab1. 2). 


\section{TABLEAU 2}

Moment d'ovulation après le début observé de l'ostrus chez des brebis normales ou traitées par un progestagène associé ou non à une injection de PMSG

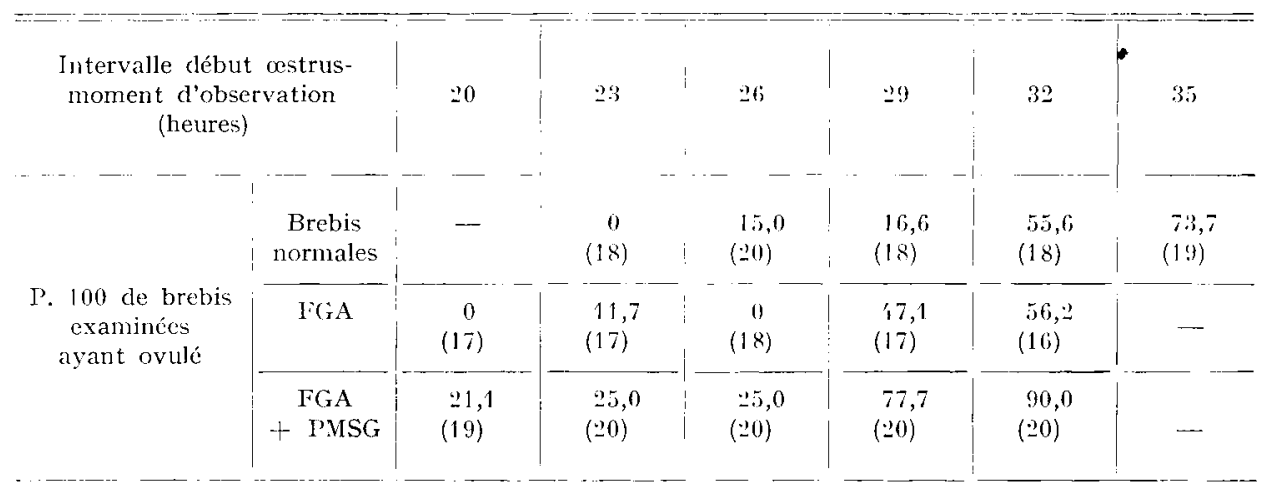

( ) Nombre d'animaux observés.

Chaque brebis n'a subi qu'un seul contrôle ovarien par colloscopie.

Mais dans ce cas, l'ajustement linéaire se révèle non satisfaisant $\left(\chi^{2}=7,32\right)$ (fig. 3). L'examen des données indique qu'il pourrait exister deux groupes d'animaux :

-- un premier groupe représentant environ $20 \mathrm{p}$. Ioo de l'échantillon examiné : la réponse à PMSG pour ces animaux est rapide puisque l'ovulation est faite moins de 20 heures après le début observé de l'œstrus ;

- un deuxième groupe pour lequel l'analyse par la méthode des probits a été possible et dont le moment moyen d'ovulation se situe à 29 h $8 \pm 0,49$ après le début observé de l'œstrus. Néanmoins, compte tenu du fait que les pourcentages ont été corrigés assez arbitrairement en retirant les $20 \mathrm{p}$. Ioo correspondant approximativement au premier groupe d'animaux, nous sommes amenés à considérer le moment moyen d'ovulation calculé par cette méthode comme une indication. En tenant compte de cette restriction, les moments moyens d'ovulation pour les trois catégories d'animaux ne sont pas différents statistiquement ce qui n'exclut pas des différences physiologiques.

\section{DISCUSSION}

\section{Estrus}

a) Cette expérience nous permet tout d'abord de confirmer ce que des expérimentations antérieures nous avaient déjà indiqué : le traitement progestatif seul est insuffisant pour provoquer l'apparition de l'oestrus chez la totalité des animaux traités pendant la période d'anœestrus ('THImonien et al., I968). Ceci peut provenir du fait que le progestagène utilisé réduit probablement la quantité de LH mobilisable lors de l'ovulation (Peilitier et Thimoniek, ig69) et que les teneurs hypophysaires 
en LH et FSH en période d'anœstrus sont déjà faibles (ThimoniER et MaulÉon, I967, I969). D'ailleurs il a été vérifié que les brebis non venues en ostrus après le traitement progestatif seul, en période d'anœstrus, n'avaient pas ovulé. Chez de tels animaux, une décharge ovulante de $\mathrm{L}_{\mathrm{H}}$ n'a pu être mise en évidence (PEILE'TIER et. Thimonier, non publié). En période d'ancestrus, une injection de PMSG est donc indispensable. Cette hormone mime l'action d'un " facteur de décharge " hypophysaire soit en agissant directement au niveau hypothalamique, soit indirectement en stimulant la croissance folliculaire des ovaires et les sécrétions ostrogéniques.

b) Aussi bien pendant la période d'anœstrus que pendant la période habituelle de reproduction, une injection de PMSG réduit considérablement l'intervalle moyen fin du traitement-début observé de l'œestrus (tabl. I). L'étalement de l'apparition est également diminué.

Cet étalement dans 1'apparition des cestrus chez les brebis recevant le progestagène seul peut provenir probablement d'un effet rémanent du progestagène. FAULKNER et HoPwOOD (I 967 ) ont montré en utilisant du FGA radioactif que l'élimination du produit est en général rapide ; cependant pour quelques animaux, l'excrétion de la radioactivité après arrêt du traitement est très longue (I $7 \mathrm{j})$. Comme nous l'avons vu précédemment, PMSG peut avoir une action soit au niveau hypothalamique, soit au niveau ovarien.

c) La période de l'année a également une influence sur l'intervalle fin du traitement-début observé de l'œstrus (tabl. I et fig. I). Cette influence de la saison sur l'apparition plus rapide de l'œstrus peut être reliée à la variation saisonnière de sensibilité, du système nerveux central, aux œstrogènes (REARDON et ROBINSON, I96I). Une variation dans la sécrétion stéroïdienne de l'ovaire n'est cependant pas à rejeter.

d) Le rythme nycthéméral est important : un grand nombre de brebis viennent en oestrus vers $I_{7}$ heures et la majorité des animaux commencent à extérioriser les manifestations de l'œstrus entre I3 heures et 22 heures.

Nous n'avons pas observé comme RobERTSON et RAKHA (I965) une deuxième période entre 4 heures et II heures, même chez les brebis témoins. Cette influence du rythme nycthéméral est accentuée lorsque une injection de PMSG est faite à la fin du traitement progestatif. Nous savons que les teneurs hypophysaires en FSH et ISCH chez les béliers sont notablement moins importantes à la fin de la photopériode claire qu'à la fin de la photopériode sombre (PELLETIER et ORTAVANT, Ig64.) Il est possible que ces deux types de constatations soient reliées, ce qui poserait le problème du rôle de la lumière comme agent de stimulation important de la libération des hormones.

Il serait donc intéressant d'étudier l'apparition de l'œestrus après modification du moment d'injection de PMSG.

\section{Ovulation}

a) Pour les brebis témoins, celles recevant le progestagène seul et celles recevant une injection de PMSG (mais dont le moment moyen d'ovulation ne diffère pas statistiquement de ceux des deux premières catégories d'animaux), le moment moyen d'ovulation se situe entre 29 h 50 et 32 heures après le dèbut de l'œstrus. ROBERTSON et RAKHA (Ig66) ont montré par des dosages hypophysaires de FSH et LH, que les décharges de ces hormones se produisent à une période bien définie après le début de 
l'œestrus. Ces observations ont été confirmées par dosage radio-immunologique de la LH plasmatique (NISWENDER et al., I968). On peut supposer que le processus est identique quel que soit le traitement reçu par les brebis d'où l'absence de signification statistique entre les moments moyens d'ovulation observés.

b) Les moments moyens d'ovulation que nous avons trouvés se situent plus tardivement par rapport au début observé de l'œestrus que ceux trouvés par ZELTOBRUKh et RAK (I964) ou PARSONS, HuNTER et RAYNer (I967) chez des brebis normales. Les conditions expérimentales indiquées par ces auteurs sont différentes des nôtres : la présence permanente ou des introductions répétées de béliers dans les lots de brebis en ostrus modifient en effet le moment de l'ovulation.

Seuls Robinson et Smith (I967) ont étudié l'ovulation après traitement progestatif. Ces auteurs n'ont trouvé aucune influence du progestagène sur le moment de l'ovulation. Nos résultats sont identiques. Mais ces auteurs n'ont pas utilisé PMSG pour l'induction de l'ovulation.

c) Il semble que l'on puisse distinguer dans la réponse à PMSG, deux types d'animaux. Un premier groupe de brebis ovulent très rapidement : moins de 20 heures après le début observé de l'œstrus quel que soit l'intervalle injection-apparition des chaleurs.

Le deuxième groupe d'animaux a, au contraire, un moment moyen d'ovulation non différent statistiquement de ceux des brebis témoins et des brebis traitées avec le progestagène seul. Cette différence entre les deux types d'animaux peut provenir d'un effet " stress ». On peut supposer que quelques brebis sont très sensibles à l'injection d'une hormone protéique (PMSG). Nous connaissons le rôle que peuvent jouer des protéines plasmatiques sur la diminution de l'acide ascorbique ovarien lors du dosage de la LH plasmatique par la méthode de PARLOw (PELLETIER, I965). Une telle hypothèse sur la sensibilité très grande de certaines brebis à un stress n'est pas à rejeter bien que les injections aient été faites par voie intramusculaire.

Rę̧u pour publication en septembre 1969.

\section{SUMMARY}

The time of ovulation in the ewe at natural cestrus and following treatment with a progestagen alone or together with PMSG.

The time of ovulation at natural ostrus was compared to that following treatment with a progestagen alone or together with PMSG in both the breeding season and during anoestrum.

A total of 93 ewes were examinated at natural cestrus, and 49 in each of the four treatment groups. Their ovaries were examinated every 3 hours from 20 to 32 or 23 to 35 hours after the onset of cestrus, by endoscopy.

Treatment with PMSG both reduced the interval between the end of progestagen treatment and the onset of ostrus, and induced a greater degree of synchronisation in cyclic and anoestrous ewes. In addition there was a slight indication that the interval between progestagen treatment and the onset of cestrus was shorter during the breeding season than during anœstrum.

The interval between the onset of cestrus and the time of natural ovulation was found to be $32,0 \pm 0,81$ hours, and $30,4 \pm 1,33$ in progestagen treated ewes. Follawing treatment with PMSG however, the interval was found to be bimodally distributed : $20 \%$ of the individuals having ovulated in less than 20 hours; the mean of $29,8 \pm 0,49$ hours for the remaining $80 \%$ being similar to that of those treated with progestagen alone. 


\section{RÉFÉRENCES BIBLIOGRAPHIQUES}

Cognte Y., Colas G., 1968. Évolution des techniques de reproduction chez les Ovins : synchronisation des chaleurs, obtention de deux agnelages par an, mise précoce à la lutte des agnelles, insémination artificielle. Pâtre, 153, r7-24.

Colas G., Cognie Y., rg68. Insémination artificiclle avec ou sans détection de chaleurs, après traitement progestatif chez la Brebis. VIe Cong. int. Reprod. anim. Insém. artif., Paris, vol II, 1407-I41o.

Faulkner L. C., Hopwood M. L., 1967. Clearance of a tritium-labeled progestin in the ewe. $J$. Anim. Sci., 26, 163-166.

Finney D. J., I962. Prohit analysis. and ed., Cambridge University Press.

Mauléon P., Dauzier L., I965. Variations de durée de l'anœestrus de lactation chez les brebis de race Ile-de-France. Ann. Biol. anim. Bioch. Biophys., 5, I3I-I43.

MCKenziE F. F., Terrill C. F., I937. Estrus, ovulation and related phenomena in the ewe. Cniv. Mo. Agric. Res. Bull. 264.

Niswender G. D., Roche J. F., Foster D. L., Midgley A. R. Jr., ig68. Radioimmunoassay of serum levels of luteinizing hormone during the cycle and early pregnancy in ewes. Proc. Soc. Exp. Biol. Med., 129, 90I-904.

Parsons S. D., Hunter G. L., Raynitr A. A., ig67. Use of probit analysis in a study of the effect of the ram on time of ovulation in the ewe. J. Reprod. Fert., 14, 7I-80.

Pelletier J. Ortavant R., ig64. Influence de la durée d'éclairement sur le contenu hypophysaire en hormones gonadotropes FSH et ICSH chez le Bélier. Ann. Biol. anim. Bioch. Biophys., 4, I7-z6.

Pelletier J,, 1965. Effet du plasma de brebis sur la décharge de LH chez la Ratte. C. R. Acad. Sci. Paris, 260, 5624-5626.

Peiletier J., Thimonier J., i969. Étude de la décharge ovulante par dosage radio-immunologique de la L.H plasmatique chez la brebis normale ou traitée par un progestagène. C. R. Acad. Sci., Paris, 268: $573-576$.

Reardon T. P., Robinson T. J., 1961. Seasonal variation in the reactivity to ostrogen of the ovariectomized ewe. Aust. J. A gric. Res., 12, 320-326.

Robertson H. A., Rakha A. M., I965. Time of onset of cestrus in the ewe. J. Reprod. Fert., 10, 27I-272.

Robertson H. A., Rakha A. M., ig66. The sequence, time and duration of the release of follicle stimulating hormone and luteinizing hormone in relation to cestrus and to ovulation in the sheep. $J$. Endocr., 35, I $77-184$.

Robinson T. J., I965. Use of progestagen impregnated sponges inserted intravaginally or subcutaneously for the control of the ostrus cycle in the sheep. Nature, 206, 39-4 I.

Robinson T. J., Smith J. F., I967. The time of ovulation after withdrawal of S. C. 9880 . rmpregnated intravaginal sponges from cyclic Merino ewes. In The control of the ovarian cycle in the sheep. Fdited by T. J. Robinson, Sydney University Press, p. 158-168.

Thimonier J., Mauléon P., 1967. Variations saisonnières des activités hypophysaires des brebis de race Ile-de-France. Coll. int. Photorégulation de la reproduction chez les Oiseaux et les Mammifères, Mont pellier, I7-22 juillet I967 (sous presse).

Thimonier J., Malléon P., Cognié Y., Ortavant R., i968. Déclenchement de l'œestrus et obtention de la gestation pendant l'anœstrus post-partum chez les brebis à l'aide d'éponges vaginales imprégnées d'acétate de fluorogestone. Ann. Zootech. 17, $257-273$.

Thimonier J., Maulíon P., r969. Variations saisonnières du comportement d'cestrus et des activités ovarienne et hypophysaire chez les Ovins. Ann. Biol. Anim. Bioch. Biophys., 9, 233-250.

Zeltobruk N. A., Rak L. P., 1964. Stimulation neurosexuelle de la fonction de reproduction des brebis (en russe). Ovtsevodstvo 10, 8-II. 